\title{
miR-126-3p and miR-451a correlate with clinicopathological features of lung adenocarcinoma: The underlying molecular mechanisms
}

\author{
QINGYONG CHEN*, HUIZHEN HU*, DEMIN JIAO*, JIE YAN, WEI XU, XIALI TANG, \\ JUN CHEN and JIAN WANG
}

Department of Respiratory Disease, The 117th Hospital of PLA, Hangzhou, Zhejiang 310013, P.R. China

Received January 11, 2016; Accepted February 18, 2016

DOI: $10.3892 / o r .2016 .4854$

\begin{abstract}
Lung cancer is the most common malignancy worldwide. This study aimed to identify miRNA biomarkers of lung adenocarcinoma and to investigate their molecular mechanisms. miRNA expression profiling of tumor tissues and adjacent normal tissues from 10 patients were detected using microarray. Differentially expressed miRNAs (DEMs) were identified, and were verified using quantitative reverse transcription-PCR. Thereafter, correlations between DEM expression and clinicopathological features were determined in 49 patients. Furthermore, Targetscan was utilized to predict target genes, among which transcription factors (TFs) were identified. The interactions among miRNAs, TFs and target genes were used to construct an miRNA-TF-target network. Totally, 11 DEMs were identified, among which two downregulated miRNAs (miR-126-3p and miR-451a) were validated. Low levels of miR-126-3p and miR-451a were associated with poor pathological stage, large tumor diameter and lymph node metastasis $(\mathrm{P}<0.05)$. Receiver operating characteristic analysis showed that both miRNAs could predict pathological stage, tumor diameter and lymph node metastasis of lung adenocarcinoma (AUC >0.65, $\mathrm{P}<0.05$ ). For miR-126-3p, 154 target genes were predicted (e.g., PLXNB2), which were enriched in 29 pathways mainly concerning apoptosis and cancer.
\end{abstract}

Correspondence to: Dr Qingyong Chen, Department of Respiratory Disease, The 117th Hospital of PLA, 14 Lingyin Road, Hangzhou, Zhejiang 310013, P.R. China

E-mail: cqyong117@163.com

*Contributed equally

Abbreviations: NSCLC, non-small cell lung cancer; DEMs, differentially expressed microRNAs; TFs, transcription factors; qRT-PCR, quantitative reverse transcription-polymerase chain reaction; ROC, receiver operating characteristics

Key words: clinicopathological features, lung adenocarcinoma, miR-126-3p, miR-451a, biomarkers
For miR-451a, 397 target genes were predicted, which were enriched in 5 pathways including 'PPAR signaling pathway'. Ten genes were co-regulated by miR-126-3p and miR-451a, e.g., TSC1. Furthermore, an miRNA-TF-target network was constructed, and a sub-network was identified, including 2 miRNAs, 15 targets, and 7 TFs. In conclusion, miR-126-3p and miR-451a predicted the severity of lung adenocarcinoma. However, the possible mechanisms explored by bioinformatics need to be further validated.

\section{Introduction}

Lung cancer is the most common malignancy worldwide (1), and approximately 1.82 million new cases and 1.56 million deaths were reported in 2012 (2). As the most common sub-type of non-small cell lung cancer (NSCLC), lung adenocarcinoma accounts for nearly $40 \%$ of cases (3). Although many advances in clinical management have been achieved recently, e.g. surgery, radiotherapy, chemotherapy, and targeted therapy, the 5-year survival rate of lung adenocarcinoma is still less than $15 \%$, in part because a majority of cases are diagnosed at a more malignant stage (4). Hence, screening individuals with high risk of developing lung adenocarcinoma at an early stage has the potential to improve clinical outcome.

MicroRNAs (miRNAs) belong to a new class of endogenous small non-coding RNAs, and they generally play key roles in regulating the translation and degradation of their target mRNAs and thus participate in many biological and pathological processes $(5,6)$. The differential expression and potential diagnostic values of miRNAs have been widely investigated in human lung adenocarcinoma $(7,8)$. For instance, Patnaik et al reported miRNAs that were differentially expressed between whole blood samples from lung adenocarcinoma patients and healthy individuals, e.g., let-7e, miR-22, miR-30a-5p, miR-185, miR-210 and miR-423-5p, using miRNA expression profiles (9). A study in a Chinese population showed that high level of miR-155 in serum specimens had a high sensitivity for diagnosing lung adenocarcinoma (10). Additionally, combinations of 4 miRNAs (miR-21, miR-486, miR-375 and miR-200b) and 7 miRNAs (miR-486, miR-126, miR-145, miR-21, miR-182, miR-200b and miR-375) in sputum were screened as marker panels to distinguish patients with stage I 
lung adenocarcinoma from healthy individuals (11). In addition, an increased level of miR-21 expression in surgical resected lung adenocarcinoma tissues was found to be related to tumor node metastasis (TNM) stage II-IV compared to stage I (12). In spite of these efforts, using aberrant miRNA expression levels to diagnose lung adenocarcinoma or predict the clinicopathological features of lung adenocarcinoma has not been fully investigated, especially in Chinese cases.

Hence, in the present study, we compared miRNA expression profiles between surgical resected lung adenocarcinoma tissues and the corresponding paired non-cancerous tissues, as well as identified the potential miRNAs with which to predict the clinicopathological features of lung adenocarcinoma. Furthermore, bioinformatics approaches such as prediction of target genes and enrichment analysis were performed to explore the underlying molecular mechanisms.

\section{Materials and methods}

Statement of ethics. All samples used in this study were obtained from the tissue bank of the 117th Hospital of the People's Liberation Army (PLA), and written informed consent was obtained from all subjects. This study was approved by the Ethics Committee of the 117th Hospital of PLA.

Patients and study design. miRNA microarray data were obtained using the tumor tissues of 10 patients (gender, 4 males and 6 females; age, $50.0 \pm 10.8$ years) with lung adenocarcinoma, as well as the paired control samples from their adjacent normal tissues. Thereafter, differentially expressed miRNAs (DEMs) were identified, and were verified using real-time quantitative reverse transcription-polymerase chain reaction (qRT-PCR) from the 10 sample pairs. Furthermore, the clinical information of the 10 patients and an additional 39 patients were collected to analyze the correlations between miRNA expression and clinicopathological features, including gender, age, pathological stage, histological grade of differentiation, tumor diameter and lymph node metastasis.

All tumor specimens used in this study were histologically classified as lung adenocarcinoma based on the World Health Organization (WHO) classification of lung tumors (13). The pathological tumor stages were determined based on the TNM classification criteria established by the International Union Against Cancer (IUAC) (14). The histological grades were classified as well/moderately/poorly differentiated (15).

RNA extraction. Total RNA was isolated using TRIzol reagent (Invitrogen, Carlsbad, CA, USA). RNA quality was determined using a Bioanalyzer (UV spectrophotometer Q3000; Quawell, San Jose, CA, USA) and RNA concentration was assessed using NanoDrop ${ }^{\mathrm{TM}} 2000$ (Thermo Scientific, Waltham, MA, USA). RNA samples were stored at $-80^{\circ} \mathrm{C}$ until used for miRNA microarray or qRT-PCR.

miRNA expression microarray. For each sample, 4-8 $\mu \mathrm{g}$ of RNA was used to perform miRNA microarray assay. Briefly, the RNA sample was size fractionated ( $<200$ nucleotides) and 3'-polyadenylated by using poly(A) polymerase. Then, an oligonucleotide tag conjugating $\mathrm{Cy} 3$ dye was ligated to the poly(A) tail for later fluorescent dye staining. Hybridization was performed overnight at $34^{\circ} \mathrm{C}$ on a $\mu$ Paraflo $^{\mathrm{TM}}$ microfluidic chip (LC Sciences, Houston, TX, USA) using 6X SSPE buffer (0.90 M NaCl, $60 \mathrm{mM} \mathrm{Na}_{2} \mathrm{HPO}_{4}, 6 \mathrm{mM}$ EDTA, pH 6.8) plus $25 \%$ formamide (16). Each of the probes on the microfluidic chip contained a chemically modified nucleotide segment complementary to a certain miRNA, as well as a long nonnucleotide molecule spacer. After washing, the chip was scanned using a laser scanner (GenePix 4000B; Molecular Devices, Sunnyvale, CA, USA) and the fluorescent images were digitized using Array-Pro image analysis software (Media Cybernetics, Carlsbad, CA, USA).

Idetification of DEMs based on the miRNA microarray. The digitized data were analyzed by first subtracting the background and then normalizing the signals using locallyweighted regression (LOWESS) filter (17). miRNAs with microarray signal $<500$ were excluded, and two-tailed pairedsample t-test (18) was used to identify the DEMs between lung adenocarcinoma and normal tissues. To obtain more potential miRNA signatures associated with lung adenocarcinoma, miRNAs with $\mathrm{P}<0.1$ and fold change $(\mathrm{FC}) \neq 1$ were considered to be DEMs in the microarray analysis.

Verification of DEMs based on qRT-PCR. Expression levels of DEMs were detected using qRT-PCR experiment. Briefly, PrimeScript ${ }^{\circledR}$ RT reagent kit (DRR037A; Takara, Tokyo, Japan) was used to reverse transcribe $40 \mathrm{ng}$ of RNA using miRNA-specific oligonucleotides. Subsequently, PCR amplification was performed using Platinum SYBR Green qPCR SuperMix-UDG kit (11733-038; Invitrogen) and the ABI StepOne Plus Real-Time PCR system (ABI PRISM ${ }^{\circledR} 7900 \mathrm{HT}$; Applied Biosystems, Foster City, CA, USA). PCR protocol was: $50^{\circ} \mathrm{C}$ for $2 \mathrm{~min}, 95^{\circ} \mathrm{C}$ for $2 \mathrm{~min}, 39$ cycles of $95^{\circ} \mathrm{C}$ for $15 \mathrm{sec}$ and $60^{\circ} \mathrm{C}$ for $30 \mathrm{sec}$. Expression levels of miRNAs in each sample were normalized to the internal control (RNU6B), and relative expression values were calculated based on the $2^{-\Delta \Delta \mathrm{Ct}}$ method. When comparing miRNA expression levels between cancer and normal groups, $\mathrm{P}<0.05$ was considered as a criterion of significance.

miRNA target prediction, enrichment analysis and miRNA-TF-target network construction. Target genes of the DEMs were predicted using TargetScan (version 6.2, http:// www.targetscan.org) (19), a target-prediction program. Based on the Kyoto Encylopedia of Genes and Genomes (KEGG) database (20), pathway enrichment analysis of the predicted target genes was carried out using Database for Annotation, Visualization and Integrated Discovery (DAVID, version 6.7, https://david.ncifcrf.gov/) (21). P-value $<0.05$ and gene count $\geq 2$ were used as the thresholds for pathway enrichment analysis. Then, transcription factors (TFs) were extracted from the target genes based on the TRANScription FACtor (TRANSFAC, version 7.0, http://www.gene-regulation.com/pub/databases. html) (22) and ENCyclopedia Of DNA Elements (ENCODE, http://encodeproject.org) (23) databases. Interactions between the protein products of the target genes were extracted from Search Tool for the Retrieval of Interacting Genes (STRING) database (24), using combination score $>0.7$ as the criterion. Finally, the miRNA-TF-target network was constructed and visualized using Cytoscape software (version 3.2.1, http:// 
A

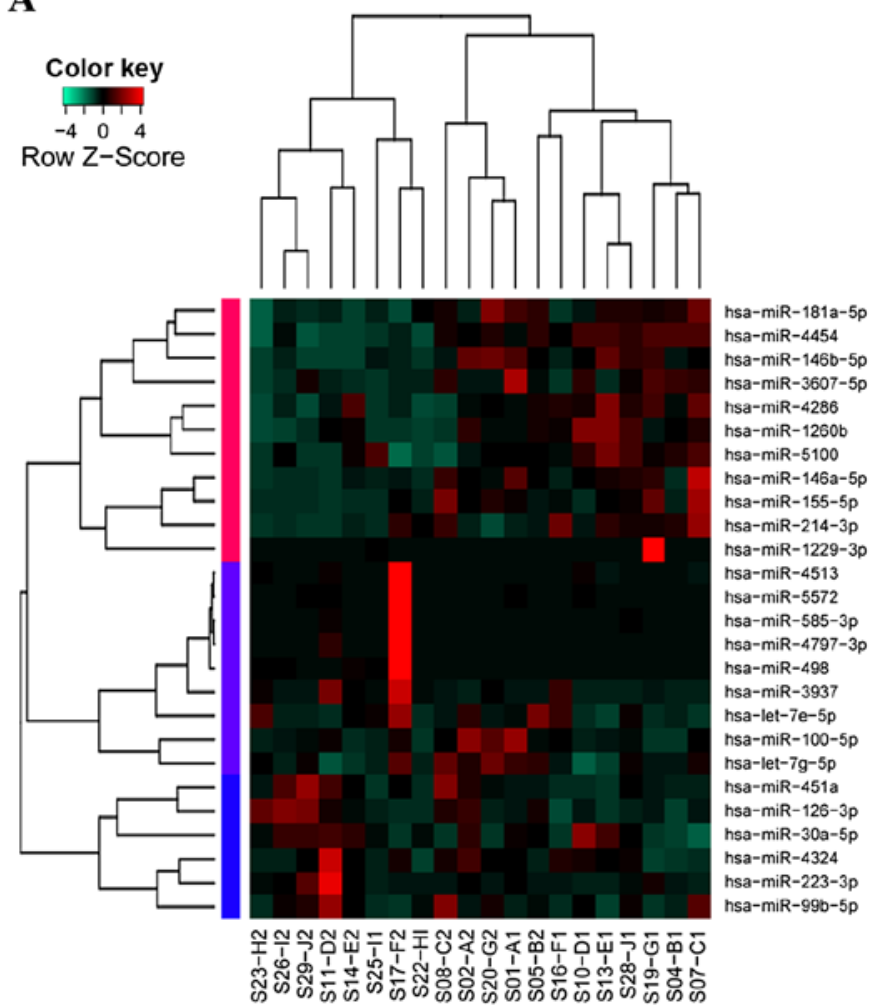

B

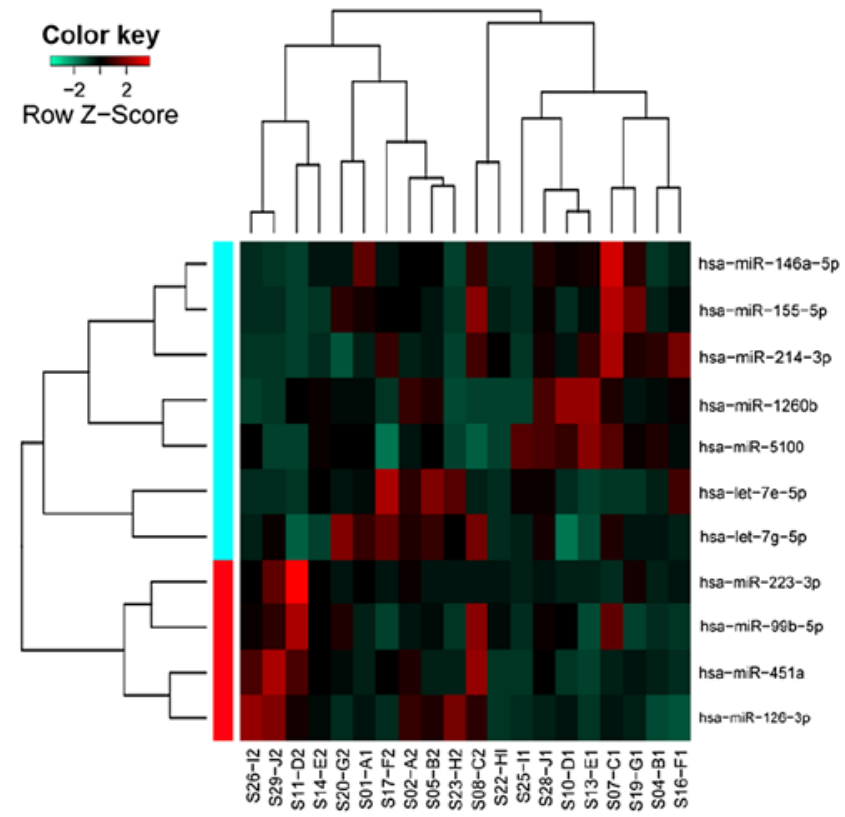

Figure 1. Unsupervised hierarchical clustering of the expression of all differentially expressed miRNAs (A) and the top 11 ones (B). Samples A1-J1 belong to the cancer group, while samples A2-J2 belong to the control group.

cytoscape.org) (25) to show the interactions among miRNAs, TFs and target genes.

Statistical analysis. All statistical analyses were performed using SPSS software (version 19.0; SPSS Inc., Chicago, IL, USA). For non-normally distributed variables, data are presented using median and interquartile range (IQR), and Mann-Whitney $U$ test was used to analyze the correlations between miRNA expression and clinicopathological features of patients with lung adenocarcinoma $(n=49)$. To further determine whether miRNA signatures have the capability of predicting clinicopathological features, receiver operating characteristics (ROC) were generated, and area under the curve and feasible threshold values were calculated. $\mathrm{P}<0.05$ was set as the cut-off criterion for statistical significance.

\section{Results}

DEMs in lung adenocarcinoma. Ten samples of lung adenocarcinoma and their paired adjacent normal tissues were analyzed via miRNA expression microarray. Overall, 119 miRNAs were differentially expressed between two groups, among them, 99 miRNAs were excluded due to their weak microarray signal (signal $<500$ ). As a result, 26 miRNAs were identified as DEMs between lung adenocarcinoma and normal tissues. Hierarchical clustering analysis showed that 26 DEMs were unable to clearly distinguish these two types of samples (sensitivity, $80 \%$; specificity, 60\%; Fig. 1A), while the top 11 DEMs displayed satisfactory sensitivity (90\%) and specificity (90\%) (Fig. 1B). Therefore, qRT-PCR was further performed for the $11 \mathrm{DEMs}$.

Validation of DEMs based on qRT-PCR. Expression levels of the 11 miRNAs were detected using qRT-PCR. Results indicated that miR-126-3p $(\mathrm{P}=1.95 \mathrm{E}-05)$ and $\mathrm{miR}-451 \mathrm{a}$ $(\mathrm{P}=1.56 \mathrm{E}-04)$ were significantly downregulated (Table I) in lung adenocarcinoma, and this was consistent with the results of the microarray analysis. In addition, changes in expression of 6 miRNAs including miR-214-3p, miR-223-3p, miR-155-5p, miR-1260b, miR-5100 and miR-146a-5p showed the same tendency, although the differences were not statistically significant (Table I; $\mathrm{P}=0.0587,0.0767,0.0856,0.218,0.247$ and 0.257 , respectively). Hence, miR-126-3p and miR-451a were defined as candidate miRNA signatures of lung adenocarcinoma, and were thus further analyzed.

Correlations between candidate miRNA signatures and clinicopathological features of lung adenocarcinoma. We next assessed the correlation of miR-126-3p and miR-451a expression (qRT-PCR) with the clinicopathological features of 49 patients with lung adenocarcinoma (gender, 24 males and 25 females; age, $59.76 \pm 12.35$ years). A total of 25 cases in pathological stage I and 24 cases in pathological stage II-IV were included, and the tumor diameter ranged from 0.8 to $12 \mathrm{~cm}$. Additionally, 32 cases were well or moderately differentiated, while 17 cases were poorly differentiated. Lymph node metastasis occurred in 22 patients. In order to show the specificity and significance of miR-126-3p and miR-451a, we 
Table I. Validation of miRNA microarray results using qRT-PCR.

\begin{tabular}{|c|c|c|c|c|c|c|}
\hline & \multicolumn{3}{|c|}{ miRNA microarray } & \multicolumn{3}{|c|}{ qRT-PCR } \\
\hline & $\mathrm{FC}$ & $\log _{2} \mathrm{FC}$ & P-value & $\mathrm{FC}$ & $\log _{2} \mathrm{FC}$ & P-value \\
\hline hsa-miR-126-3p $\mathrm{p}^{\mathrm{a}, \mathrm{b}}$ & 0.46 & -1.12 & 4.68E-04 & 0.29 & -0.56 & $1.95 \mathrm{E}-05$ \\
\hline hsa-miR-451a a,b & 0.23 & -2.11 & $1.12 \mathrm{E}-03$ & 0.26 & -0.51 & $1.56 \mathrm{E}-04$ \\
\hline hsa-miR-99b-5p & 0.73 & -0.45 & $3.39 \mathrm{E}-02$ & 1.51 & 1.68 & $2.32 \mathrm{E}-02$ \\
\hline hsa-miR-214-3pa & 1.73 & 0.79 & $2.59 \mathrm{E}-03$ & 2.53 & 0.75 & $5.87 \mathrm{E}-02$ \\
\hline hsa-miR-223-3p ${ }^{a}$ & 0.48 & -1.06 & $6.46 \mathrm{E}-02$ & 0.76 & -2.53 & 7.67E-02 \\
\hline hsa-miR-155-5p $\mathrm{p}^{\mathrm{a}}$ & 1.53 & 0.61 & 4.45E-02 & 2.26 & 0.85 & $8.56 \mathrm{E}-02$ \\
\hline hsa-let-7g-5p & 0.62 & -0.69 & $1.27 \mathrm{E}-02$ & 1.19 & 3.98 & $1.56 \mathrm{E}-01$ \\
\hline hsa-let-7e-5p & 0.55 & -0.87 & $9.92 \mathrm{E}-02$ & 1.31 & 2.57 & $1.76 \mathrm{E}-01$ \\
\hline hsa-miR-1260b & 2.17 & 1.12 & $3.12 \mathrm{E}-02$ & 1.42 & 1.98 & $2.18 \mathrm{E}-01$ \\
\hline hsa-miR-5100a & 1.77 & 0.82 & 4.97E-03 & 1.50 & 1.71 & $2.47 \mathrm{E}-01$ \\
\hline hsa-miR-146a-5p ${ }^{a}$ & 1.97 & 0.98 & $6.16 \mathrm{E}-02$ & 2.34 & 0.82 & $2.57 \mathrm{E}-01$ \\
\hline
\end{tabular}

Table II. Correlation between clinicopathological features and expression levels of miR-126-3p, miR-451a and miR-214-3p in 49 patients with lung adenocarcinoma.

\begin{tabular}{|c|c|c|c|c|c|c|c|}
\hline \multirow[b]{2}{*}{ Parameter } & \multirow[b]{2}{*}{$\mathrm{N}(\%)$} & \multicolumn{2}{|c|}{ miR-126-3p } & \multicolumn{2}{|c|}{ miR-451a } & \multicolumn{2}{|c|}{$\operatorname{miR}-214-3 p$} \\
\hline & & Median (IQR) & $\mathrm{P}$-value & Median (IQR) & P-value & Median (IQR) & P-value \\
\hline \multicolumn{8}{|l|}{ Gender } \\
\hline Male & $24(49)$ & $11.96(6.77,39.59)$ & 0.603 & $3.45(1.18,10.16)$ & 0.689 & $1.00(0.646,1.88)$ & 0.203 \\
\hline Female & $25(51)$ & $22.46(7.07,33.67)$ & & $3.45(1.25,9.38)$ & & $1.71(0.77,2.23)$ & \\
\hline \multicolumn{8}{|l|}{ Age (years) } \\
\hline$\leq 60$ & $25(51)$ & $17.99(6.39,34.95)$ & 0.795 & $3.45(1.21,9.28)$ & 0.920 & $1.61,(0.67,2.18)$ & 0.484 \\
\hline$>60$ & $24(49)$ & $16.76(7.62,35.25)$ & & $3.40(1.23,11.10)$ & & $1.13,(0.65,1.92)$ & \\
\hline \multicolumn{8}{|l|}{ Pathological stage } \\
\hline I & $25(51)$ & $24.45(9.26,57.34)$ & $0.010^{\mathrm{a}}$ & $4.71(3.29,13.31)$ & $0.004^{\mathrm{b}}$ & $1.79(0.65,2.53)$ & 0.379 \\
\hline II-IV & $24(49)$ & $8.81(5.50,22.14)$ & & $1.67(0.90,7.15)$ & & $1.23(0.67,1.81)$ & \\
\hline \multicolumn{8}{|l|}{$\begin{array}{l}\text { Histologic grade of } \\
\text { differentiation }\end{array}$} \\
\hline Well/moderately & $32(65)$ & $16.81(6.98,29.06)$ & 0.900 & $3.52(1.60,9.43)$ & 0.721 & $1.53(0.69,2.10)$ & 0.366 \\
\hline Poorly & $17(37)$ & $17.87(6.23,46.71)$ & & $2.47(1.04,10.69)$ & & $1.17(0.59,1.92)$ & \\
\hline \multicolumn{8}{|c|}{ Tumor diameter $(\mathrm{cm})$} \\
\hline$\leq 3$ & $29(59)$ & $23.54(8.53,56.01)$ & $0.038^{\mathrm{a}}$ & $4.09(2.10,13.52)$ & $0.030^{\mathrm{a}}$ & $1.53(0.69,2.08)$ & 0.502 \\
\hline$>3$ & $20(41)$ & $10.60(4.91,23.03)$ & & $2.40(0.87,7.46)$ & & $1.32(0.56,1.92)$ & \\
\hline \multicolumn{8}{|c|}{ Lymph node metastasis } \\
\hline Negative & $27(55)$ & $23.54(8.86,53.03)$ & $0.023^{\mathrm{a}}$ & $4.28(3.26,11.16)$ & $0.003^{\mathrm{b}}$ & $1.79(0.61,2.25)$ & 0.688 \\
\hline Positive & $22(45)$ & $8.82(5.87,21.61)$ & & $1.58(0.83,5.63)$ & & $1.24(0.68,1.80)$ & \\
\hline
\end{tabular}

${ }^{\mathrm{a}} \mathrm{P}<0.05 ;{ }^{\mathrm{b}} \mathrm{P}<0.01$. The expression levels of miRNAs were detected by qRT-PCR and normalized to the internal control (U6). Data of the expression levels are expressed as median (IQR). Comparisons were performed using the Mann-Whitney U test. IQR, interquartile range; qRT-PCR, quantitative reverse transcription-polymerase chain reaction.

utilized a negative control, miR-214-3p, which had a tendency to be significant in the qRT-PCR validation.
As a consequence, no significant correlation was observed between the expression levels of miR-126-3p (or miR-451a) and 

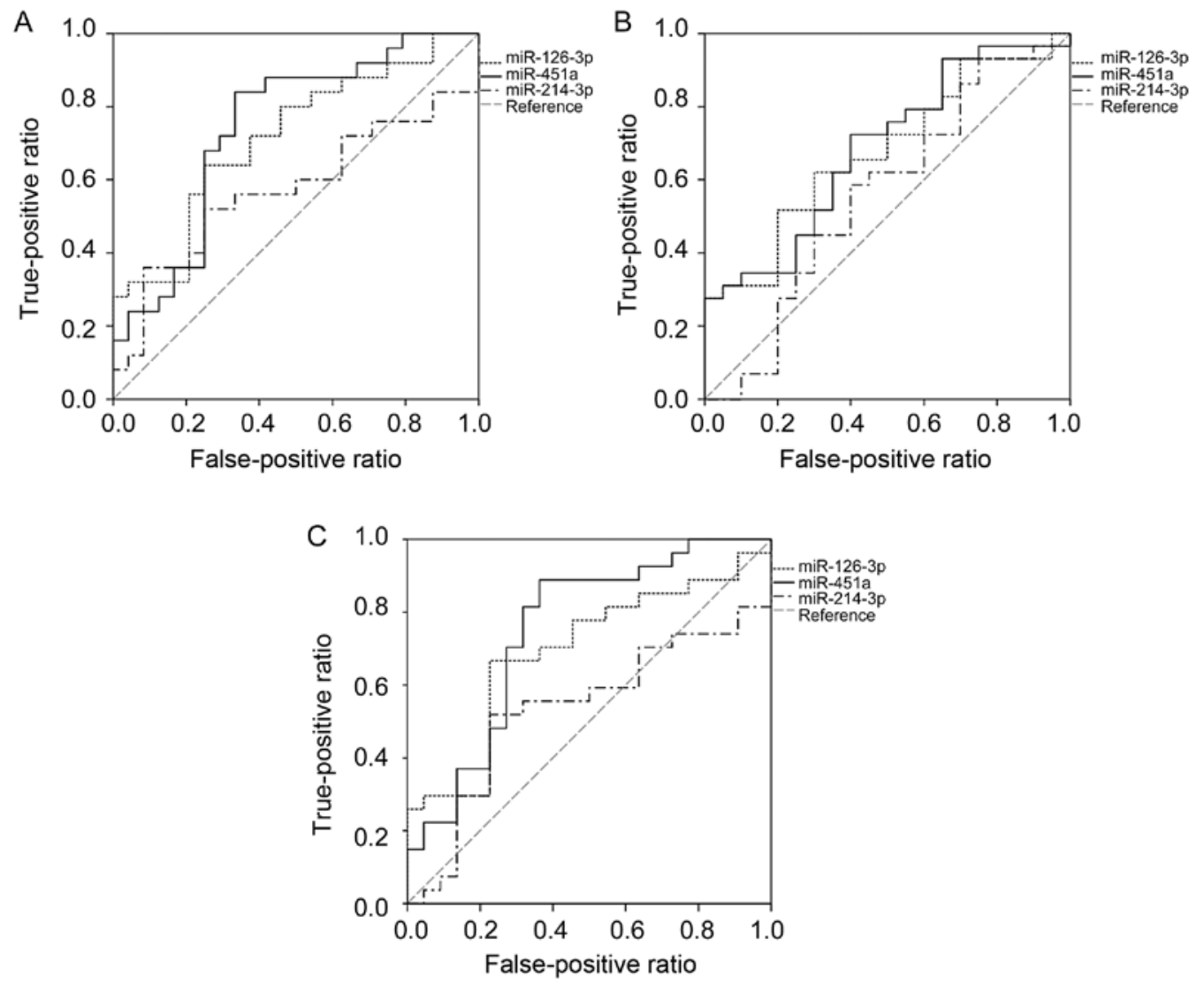

Figure 2. ROC curves of miR-126-3p, miR-451a and miR-214-3p for assessment of clinical stage (A), tumor diameter (B) and lymph node metastasis (C). ROC, receiver operating characteristics.

3 clinicopathological features including gender, age and histological grade of differentiation ( $\mathrm{P}>0.05$; Table II). However, lower expression levels of miR-126-3p and miR-451a were detected in pathological stage II-IV cases when compared with stage I cases ( $\mathrm{P}=0.010$ and 0.004 , respectively), in cases with tumor diameter $>3 \mathrm{~cm}$ when compared with those with tumor diameter $\leq 3 \mathrm{~cm}$ ( $\mathrm{P}=0.038$ and 0.030 , respectively), as well as in cases with lymph node metastasis when compared with those without lymph node metastasis $(\mathrm{P}=0.023$ and 0.003 , respectively) (Table II). Additionally, no correlation was observed between miR-214-3p expression level and any tested clinicopathological feature $(\mathrm{P}>0.05)$.

The ROC results suggested that both miR-126-3p and miR-451a had the capability to predict pathological stage, tumor diameter and occurrence of lymph node metastasis (Fig. 2). The optimal cut-off values of miR-126-3p were 20.29, 17.93 and 18.44 for predicting pathological stage $(\mathrm{AUC}=0.715, \mathrm{P}=0.010)$, tumor diameter $(\mathrm{AUC}=0.676, \mathrm{P}=0.038)$ and lymph node metastasis $(\mathrm{AUC}=0.690, \mathrm{P}=0.023)$. The corresponding sensitivity and specificity were 64 and 75\%, 62 and $70 \%$, and 67 and $77 \%$, respectively. At a cut-off value of 3.02, 3.02 and 2.40, miR-451a showed the best potential to predict pathological stage $(\mathrm{AUC}=0.742, \mathrm{P}=0.004)$, tumor diameter $(\mathrm{AUC}=0.684$, $\mathrm{P}=0.030)$ and lymph node metastasis $(\mathrm{AUC}=0.749, \mathrm{P}=0.003)$. The corresponding sensitivity and specificity were 84 and $67 \%, 72$ and $60 \%$, and 89 and $64 \%$, respectively. In addition, there was no difference between miR-126-3p and miR-451a in the capability of predicting pathologic stage $(\mathrm{P}=0.317)$, tumor diameter $(\mathrm{P}=0.090)$ or lymph node metastasis $(\mathrm{P}=0.438)$.
miRNA-TF-target network associated with lung adenocarcinoma and pathway enrichment analysis of target genes. Totally, 154 and 397 target genes of miR-126-3p and miR-451a were predicted, respectively, and 10 genes were co-regulated by miR-126-3p and miR-451a, including AMMECR1L, FBXO33, GATAD2B, HIP1, KCMF1, KIAA1456, PCDH7, SAMD12, TSC1 and ZADH2. Target genes of miR-126-3p were significantly enriched in 29 KEGG pathways such as 'apoptosis', 'prostate cancer', 'focal adhesion', 'mTOR signaling pathway', 'endometrial cancer' and 'non-small cell lung cancer' (the top 15 pathways are shown in Table III), while target genes of miR-451a were markedly enriched in 5 KEGG pathways such as 'PPAR signaling pathway' (e.g., PPARA) (Table III).

Furthermore, 14 TFs and 181 protein-protein interactions were found among the target genes of miR-126-3p and miR-451a. Then, an miRNA-TF-target network was constructed (Fig. 3), containing 2 miRNAs, 7 TFs, 148 targets, as well as 150 miRNA-target pairs, 181 protein-protein pairs, and 21 TF-target pairs. For instance, PLXNB2 was targeted by miR-126-3p, PPARA was targeted by miR-451a, and TSC1 was targeted by both miR-126-3p and miR-451a. In addition, a sub-network was identified in the miRNA-TF-target network, including 2 miRNAs, 15 targets, and 7 TFs (Fig. 4). Only target genes which were targeted by both miRNAs and TFs were included in this sub-network. The regulations between miRNAs and target genes $(n=22)$ as well as regulations between TFs and target genes $(n=19)$ were clearly revealed in the sub-network. 
Table III. Pathway enrichment analysis of the target genes of miR-126-3p and miR-451a.

\begin{tabular}{|c|c|c|c|c|c|}
\hline miRNAs & ID & Terms & Count & P-value & Gene symbols \\
\hline \multirow[t]{15}{*}{$\operatorname{miR}-126-3 p$} & 4722 & Neurotrophin signaling pathway & 8 & $2.00 \mathrm{E}-05$ & $\begin{array}{l}\text { BCL2, CRK, FOXO3, FRS2, IRS1, IRS2, } \\
\text { PIK3CD, PIK3R2 }\end{array}$ \\
\hline & 4960 & $\begin{array}{l}\text { Aldosterone-regulated sodium } \\
\text { reabsorption }\end{array}$ & 5 & 3.92E-05 & IRS1, IRS2, KCNJ1, PIK3CD, PIK3R2 \\
\hline & 4210 & Apoptosis & 6 & $1.44 \mathrm{E}-04$ & $\begin{array}{l}\text { BCL2, DFFB, PIK3CD, PIK3R2, PPP3CB, } \\
\text { TNFRSF10B }\end{array}$ \\
\hline & 4930 & Type II diabetes mellitus & 4 & $9.89 \mathrm{E}-04$ & IRS1, IRS2, PIK3CD, PIK3R2 \\
\hline & 4910 & Insulin signaling pathway & 6 & $1.70 \mathrm{E}-03$ & CRK, IRS1, IRS2, PIK3CD, PIK3R2, TSC1 \\
\hline & 4730 & Long-term depression & 4 & $4.00 \mathrm{E}-03$ & GNA13, IGF1R, NOS1, PLA2G12A \\
\hline & 4370 & VEGF signaling pathway & 4 & $5.37 \mathrm{E}-03$ & PIK3CD, PIK3R2, PLA2G12A, PPP3CB \\
\hline & 533 & $\begin{array}{l}\text { Glycosaminoglycan } \\
\text { biosynthesis-keratan sulfate }\end{array}$ & 2 & 8.37E-03 & B4GALT4, CHST6 \\
\hline & 5215 & Prostate cancer & 4 & $9.35 \mathrm{E}-03$ & BCL2, IGF1R, PIK3CD, PIK3R2 \\
\hline & 4510 & Focal adhesion & 6 & $1.05 \mathrm{E}-02$ & BCL2, CRK, IGF1R, PARVA, PIK3CD, PIK3R2 \\
\hline & 4150 & mTOR signaling pathway & 3 & $1.24 \mathrm{E}-02$ & PIK3CD, PIK3R2, TSC1 \\
\hline & 5213 & Endometrial cancer & 3 & $1.24 \mathrm{E}-02$ & FOXO3, PIK3CD, PIK3R2 \\
\hline & 5014 & Amyotrophic lateral sclerosis (ALS) & 3 & $1.31 \mathrm{E}-02$ & BCL2, NOS1, PPP3CB \\
\hline & 5223 & Non-small cell lung cancer & 3 & $1.38 \mathrm{E}-02$ & FOXO3, PIK3CD, PIK3R2 \\
\hline & 5210 & Colorectal cancer & 3 & 1.99E-02 & BCL2, PIK3CD, PIK3R2 \\
\hline \multirow[t]{5}{*}{$\operatorname{miR}-451 \mathrm{a}$} & 3320 & PPAR signaling pathway & 5 & $1.21 \mathrm{E}-02$ & ACADL, ADIPOQ, CYP8B1, GK, PPARA \\
\hline & 561 & Glycerolipid metabolism & 4 & $1.66 \mathrm{E}-02$ & AKR1B1, DAK, GK, LIPG \\
\hline & 51 & Fructose and mannose metabolism & 3 & $3.35 \mathrm{E}-02$ & AKR1B1, C12orf5, PMM2 \\
\hline & 360 & Phenylalanine metabolism & 2 & 4.34E-02 & ALDH3B2, MIF \\
\hline & 620 & Pyruvate metabolism & 3 & 4.38E-02 & ACACA, ACYP2, AKR1B1 \\
\hline
\end{tabular}

The top 15 pathways are shown for miR-126-3p while all pathways are shown for miR-451a.

\section{Discussion}

A number of studies have directly profiled miRNA expression in lung cancers, and some miRNAs have been identified for cancer diagnosis and prognosis. However, limited studies have focused on predicting clinicopathological features of lung cancers (26-28), especially lung adenocarcinoma. In this study, we screened the miRNA signatures using miRNA expression profiling and qRT-PCR and identified two miRNAs (miR-126-3p and miR-451a), which were significantly correlated with pathological stage, tumor diameter and lymph node metastasis. In addition, ROC analysis showed that both miR-126-3p and miR-451a were significantly correlated with pathological stage, tumor diameter and occurrence of lymph node metastasis.

Jusufovic et al reported that the miR-126 level was lower in NSCLC (including squamous and adenocarcinoma) tissues in comparison with normal controls (29). However, it is unknown whether miR-126 is a special signature for the severity of lung adenocarcinoma. We also found that miR-126-3p was downregulated in lung adenocarcinoma tissues, and low expression of miR-126-3p was associated with large tumor size, as well as high pathological stage. Liu et al showed that exogenous expression of miR-126 could inhibit the proliferation of human lung adenocarcinoma epithelial cells and reduce the tumor weight in mice (30). Therefore, we proposed that the downregulation of miR-126-3p in lung adenocarcinoma could promote tumor cell proliferation and consequently increase tumor severity.

Bioinformatics analysis was conducted to preliminarily explore the possible molecular mechanism underlying the effects of miR-126-3p on lung adenocarcinoma. A total of 154 genes were predicted to be regulated by miR-126-3p, and these target genes were mainly enriched in cancer-related pathways, e.g., 'apoptosis', 'prostate cancer', 'focal adhesion', 'endometrial cancer' and 'non-small cell lung cancer'. Especially, miR-126-3p was predicted to target plexin B2 (PLXNB2), which plays a role in RhoA activation (31). Reportedly, RhoA was found to promote cell proliferation in breast cancer and esophageal squamous cell carcinoma cell lines $(32,33)$. Additionally, Pillé et al found that miR-126 could suppress cell proliferation via inhibiting the RhoA/ROCK signaling pathway in colon cancer cell lines (34). Hence, we supposed that a similar mechanism may exist in lung adenocarcinoma, i.e., miR-126-3p could restrain cell proliferation via inhibiting PLXNB2 expression and the subsequent RhoA/ROCK signaling pathway. In lung adenocarcinoma, miR-126-3p was significantly downregulated, and thus its target PLXNB2 might be upregulated, resulting in increased tumor cell proliferation and tumor severity. 


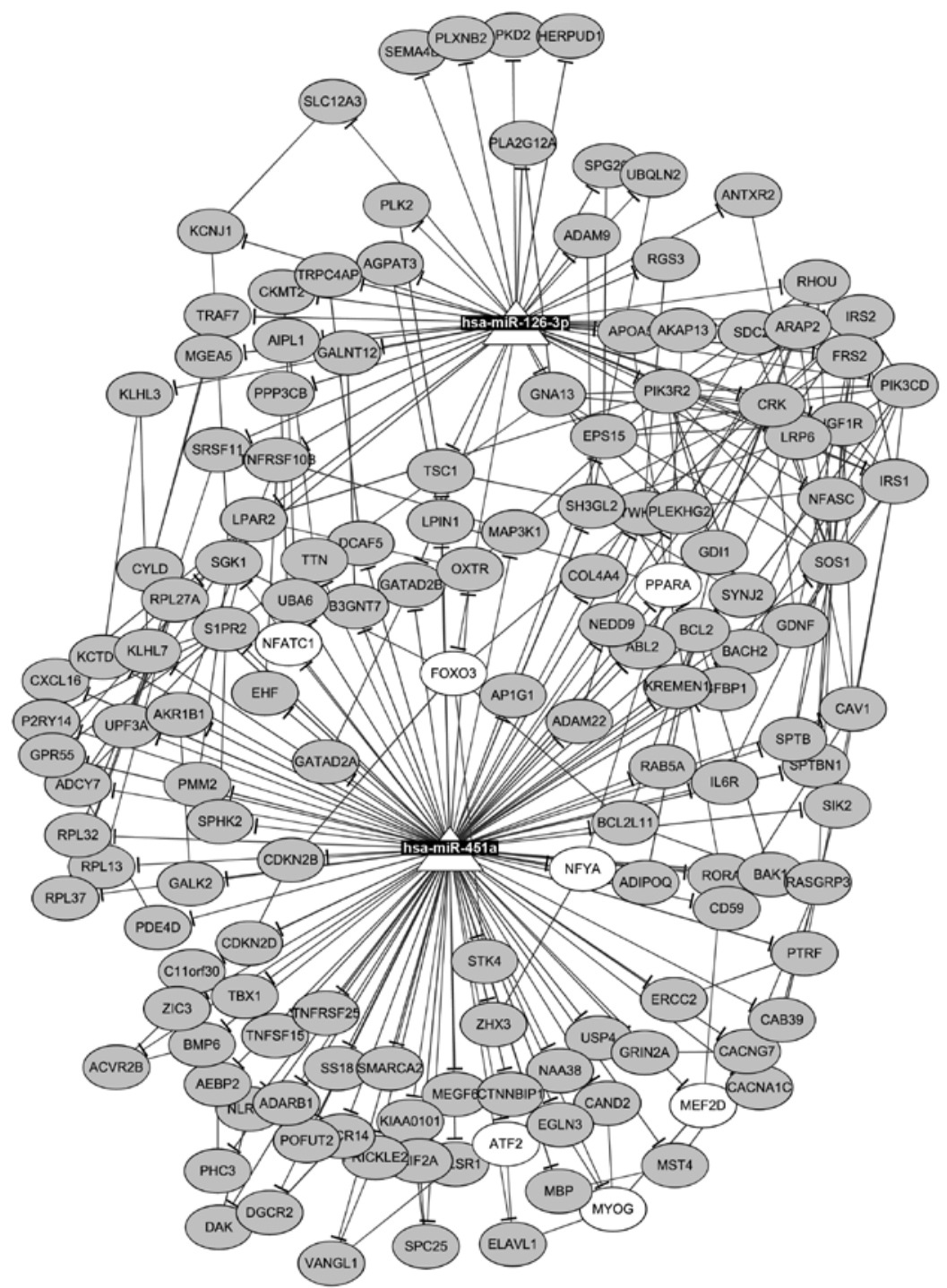

Figure 3. miRNA-TF-target network of miR-126-3p and miR-451a. White triangles represent miRNAs, white circles represent TFs, and grey circles represent target genes. Regulatory interactions between miRNAs and their target genes were predicted using TargetScan, protein-protein interactions between target genes were obtained from STRING database, and regulations between TFs and their target genes were extracted from TRANSFAC and ENCODE databases. TFs, transcription factors; miRNAs, microRNAs.

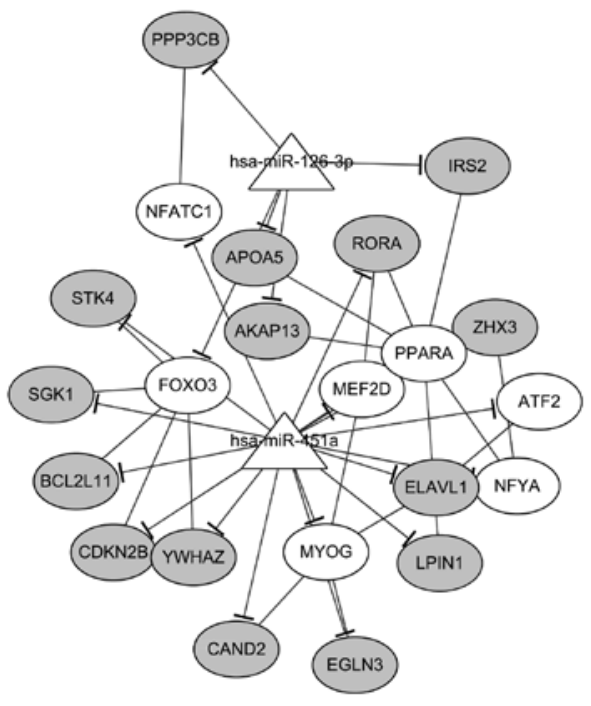

Figure 4. miRNA-TF-target sub-network of of miR-126-3p and miR-451a White triangles represent miRNAs, white circles represent TFs, and grey circles represent target genes. TFs, transcription factors; miRNAs, microRNAs.
Moreover, we found that decreased expression of miR-126-3p was related to the occurrence of lymph node metastasis. Reportedly, RhoA is a key regulator of tumor cell motility (35), and its activation is associated with PLXNB2, a target of miR-126-3p. In addition, over-activation of RhoA promotes non-physiological breakage of cell-cell and cell-substrate contacts partly by regulating cytoskeletal activity, including actin assembly, microtubule dynamics and myosin II-dependent contractility of the actin-rich cortex (36). Hence, PLXNB2-activated RhoA signaling might be involved in the role of miR-126-3p in lymph node metastasis of lung adenocarcinoma.

miR-451a has been confirmed as a downregulated miRNA in lung cancer by using meta-analyses $(37,38)$. Wang et al reported that low expression of miR-451 in NSCLC patients was associated with poor tumor differentiation, high pathological stage and occurrence of lymph node metastasis, as well as reduced overall survival of NSCLC patients (39). In the present study, it was found that low expression of miR-451a 
was significantly associated with high pathological stage II-IV and lymph node metastasis of lung adenocarcinoma, and this was consistent with a previous study (39). Furthermore, the ROC results suggested that miR-451a could sensitively and specifically predict pathological stage, tumor diameter and occurrence of lymph node metastasis of lung adenocarcinoma, indicating that miR-451a is a potential signature and biomarker of lung adenocarcinoma. Reportedly, miR-451 suppressed the in vitro proliferation of NSCLC cells and NSCLC development via downregulating ras-related protein 14 (RAB14). In this study, RAB14 was also predicted as a target of miR-451, showing the accuracy of the TargetScan tool. Moreover, the target genes of miR-451a were most significantly enriched in the peroxisome proliferator-activated receptor (PPAR) signaling pathway, e.g., PPARA (PPAR $\alpha$ ). Although there is no direct evidence for the involvement of PPAR $\alpha$ in metastasis, downregulation of PPAR $\gamma$ contributes to gastric carcinoma and lymph node metastasis (40). Reportedly, PPAR $\gamma$ is strongly expressed in lung cancer tissues (41), and its agonists inhibit human lung cancer cell growth via inducting apoptosis (42). Hence, miR-451a might regulate the progression and lymph node metastasis of lung adenocarcinoma via modulating the PPAR signaling pathway.

In the present study, tuberous sclerosis 1 (TSC1) was co-regulated by miR-126-3p and miR-451a. Reportedly, loss of heterozygosity in TSC1-gene-region on chromosome 9q34 is frequently observed in lung adenocarcinoma (43), and Tsc1 loss can synergize with Kras mutation to enhance lung tumorigenesis in mouse via activating mammalian target of rapamycin (mTOR) (44). In this study, miR-126-3p and miR-451a were both downregulated in lung adenocarcinoma, reducing the suppression on TSLC1 expression. This result was inconsistent with previous studies $(43,44)$, indicating that TSC1 expression might also be regulated by other miRNAs or TFs.

miR-214 is also a cancer-related miRNA but functions as an oncogene or a tumor suppressor in different types of cancers. miR-214 is downregulated in cervical cancer and negatively regulates HeLa cell proliferation (45), while upregulated miR-214 has been reported in pancreatic cancer (46) and gastric cancer (47) and is associated with poor overall survival of patients. Higher expression level of miR-214 was also found in lung cancer tissues vs. non-cancerous lung tissues according to microarray data, which, however, was not validated by qRT-PCR (48). Ishimura et al reported that miR-214 is overexpressed in cancerous lung tissues from primary NSCLC (49). We firstly reported that although the expression level of miR-214 was slightly higher in lung adenocarcinoma when compared with that in the paired noncancerous tissues, the difference was not significant. These findings further indicate the different functions of miR-214 in different types of lung carcinoma.

In conclusion, in this study, miR-126-3p and miR-451a were found to be significantly downregulated in human lung adenocarcinoma. The low levels of miR-126-3p and miR-451a in lung adenocarcinoma were significantly correlated with pathological stage, tumor diameter and occurrence of lymph node metastasis. In addition, miR-126-3p and miR-451a might play their roles in lung adenocarcinoma via regulating the expression of target genes, e.g., PLXNB2 and PPARA. In our future study, the target relationships between miR-126-3p and
PLXNB2, as well as miR-451a and PPARA will be validated using luciferase assay, and their roles will be further investigated using shRNA silencing and vector overexpressing technologies.

\section{Acknowledgements}

This study was supported by Science and Technology Plan Project of Hangzhou City (20130633829, 20140633840), Public Welfare Project of Zhejiang Science and Technology Department (2013C33209, 2014C33277) and Natural Science Foundation of Zhejiang Province (LY12H16001).

\section{References}

1. Teh E and Belcher E: Lung cancer: Diagnosis, staging and treatment. Surgery 32: 242-248, 2014.

2. Stewart BW and Wild CP (eds): World Cancer Report 2014. World Health Organization, Lyon, 2014.

3. Lu C, Onn A and Vaporciyan A: 78: Cancer of the Lung. Holland-Frei Cancer Medicine. 8th edition. People's Medical Publishing House, 2010.

4. Jemal A, Siegel R, Ward E, Murray T, Xu J, Smigal C and Thun MJ: Cancer statistics, 2006. CA Cancer J Clin 56: 106-130, 2006.

5. Frazier TP and Zhang B: Identification of plant microRNAs using expressed sequence tag analysis. In: Plant Reverse Genetics. Springer, pp13-25, 2011.

6. Schneider MR: MicroRNAs as novel players in skin development, homeostasis and disease. Br J Dermatol 166: 22-28, 2012.

7. Fu SW, Chen L and Man YG: miRNA biomarkers in breast cancer detection and management. J Cancer 2: 116-122, 2011

8. Nicolas FE, Lopez-Gomollon S, Lopez-Martinez AF and Dalmay T: Silencing human cancer: Identification and uses of microRNAs. Recent Pat Anticancer Drug Discov 6: 94-105, 2011.

9. Patnaik SK, Yendamuri S, Kannisto E, Kucharczuk JC, Singhal S and Vachani A: MicroRNA expression profiles of whole blood in lung adenocarcinoma. PLoS One 7: e46045, 2012.

10. Gao F, Chang J, Wang H and Zhang G: Potential diagnostic value of miR-155 in serum from lung adenocarcinoma patients. Oncol Rep 31: 351-357, 2014.

11. Yu L, Todd NW, Xing L, Xie Y, Zhang H, Liu Z, Fang H, Zhang J, Katz RL and Jiang F: Early detection of lung adenocarcinoma in sputum by a panel of microRNA markers. Int J Cancer 127: 2870-2878, 2010 .

12. Saito M, Schetter AJ, Mollerup S, Kohno T, Skaug V, Bowman ED, Mathé EA, Takenoshita S, Yokota J, Haugen A, et al: The association of microRNA expression with prognosis and progression in early-stage, non-small cell lung adenocarcinoma: A retrospective analysis of three cohorts. Clin Cancer Res 17: 1875-1882, 2011.

13. Travis WD, Brambilla W, Mueller-Hermelink HK and Harris CC: World Health Organization classification of tumors: Pathology and Genetics of Tumors of the Lung, Pleura, Thymus and Heart. IARC Press, Lyon, 2004.

14. Sobin LH, Gospodarowicz MK and Wittekind C (eds): TNM Classification of Malignant Tumours. 7th edition. Wiley-Blackwell, 2009.

15. Ohtsuka T, Nomori H, Watanabe K, Kaji M, Naruke T, Suemasu K and Uno K: Prognostic significance of [(18)F]fluorodeoxyglucose uptake on positron emission tomography in patients with pathologic stage I lung adenocarcinoma. Cancer 107: 2468-2473, 2006.

16. Gao X, Gulari E and Zhou X: In situ synthesis of oligonucleotide microarrays. Biopolymers 73: 579-596, 2004.

17. Bolstad BM, Irizarry RA, Astrand M and Speed TP: A comparison of normalization methods for high density oligonucleotide array data based on variance and bias. Bioinformatics 19: 185-193, 2003.

18. Streichert T, Otto B and Lehmann U: MicroRNA profiling using fluorescence-labeled beads: data acquisition and processing. In: MicroRNA and Cancer. Springer, pp253-268, 2011.

19. Lewis BP, Burge CB and Bartel DP: Conserved seed pairing, often flanked by adenosines, indicates that thousands of human genes are microRNA targets. Cell 120: 15-20, 2005. 
20. Kanehisa M and Goto S: KEGG: Kyoto encyclopedia of genes and genomes. Nucleic Acids Res 28: 27-30, 2000.

21. Huang DW, Sherman BT, Tan Q, Collins JR, Alvord WG, Roayaei J, Stephens R, Baseler MW, Lane HC and Lempicki RA The DAVID Gene Functional Classification Tool: A novel biological module-centric algorithm to functionally analyze large gene lists. Genome Biol 8: R183, 2007.

22. Matys V, Kel-Margoulis OV, Fricke E, Liebich I, Land S, Barre-Dirrie A, Reuter I, Chekmenev D, Krull M, Hornischer K, et $a l$ : TRANSFAC and its module TRANSCompel: Transcriptional gene regulation in eukaryotes. Nucleic Acids Res 34: D108-D110, 2006.

23. Rosenbloom KR, Sloan CA, Malladi VS, Dreszer TR, Learned K, Kirkup VM, Wong MC, Maddren M, Fang R, Heitner SG, et al: ENCODE data in the UCSC Genome Browser: Year 5 update. Nucleic Acids Res 41: D56-D63, 2013.

24. Franceschini A, Szklarczyk D, Frankild S, Kuhn M, Simonovic M, Roth A, Lin J, Minguez P, Bork P, von Mering C, et al: STRING v9.1: Protein-protein interaction networks, with increased coverage and integration. Nucleic Acids Res 41: D808-D815, 2013.

25. Smoot ME, Ono K, Ruscheinski J, Wang P-L and Ideker T: Cytoscape 2.8: New features for data integration and network visualization. Bioinformatics 27: 431-432, 2011.

26. Barshack I, Lithwick-Yanai G, Afek A, Rosenblatt K, Tabibian-Keissar H, Zepeniuk M, Cohen L, Dan H, Zion O, Strenov Y, et al: MicroRNA expression differentiates between primary lung tumors and metastases to the lung. Pathol Res Pract 206: 578-584, 2010.

27. Raponi M, Zhang Y, Yu J, Chen G, Lee G, Taylor JM, Macdonald J, Thomas D, Moskaluk C, Wang Y, et al: Gene expression signatures for predicting prognosis of squamous cell and adenocarcinomas of the lung. Cancer Res 66: 7466-7472, 2006.

28. Kim MK, Jung SB, Kim JS, Roh MS, Lee JH, Lee EH and Lee HW: Expression of microRNA miR-126 and miR-200c is associated with prognosis in patients with non-small cell lung cancer. Virchows Arch 465: 463-471, 2014.

29. Jusufovic E, Keser D, Zukic E, Sejdinovic R and Mrsic D: Downregulated anti-angiogenic miR-19a, miR-126 and let-7b in non-small lung cancer have poor but different prognostic values in squamous and adenocarcinoma subtypes. Eur Respir J 42 4642, 2013

30. Liu B, Peng XC, Zheng X-L, Wang J and Qin YW: miR-126 restoration down-regulate VEGF and inhibit the growth of lung cance cell lines in vitro and in vivo. Lung Cancer 66: 169-175, 2009.

31. Perrot V, Vázquez-Prado J and Gutkind JS: Plexin B regulates Rho through the guanine nucleotide exchange factors leukemia-associated Rho GEF (LARG) and PDZ-RhoGEF. J Biol Chem 277: 43115-43120, 2002.

32. Li N, Tang A, Huang S, Li Z, Li X, Shen S, Ma J and Wang X: miR-126 suppresses colon cancer cell proliferation and invasion via inhibiting RhoA/ROCK signaling pathway. Mol Cell Biochem 380: 107-119, 2013

33. Faried A, Faried LS, Kimura H, Nakajima M, Sohda M, Miyazaki T, Kato H, Usman N and Kuwano H: RhoA and RhoC proteins promote both cell proliferation and cell invasion of human oesophageal squamous cell carcinoma cell lines in vitro and in vivo. Eur J Cancer 42: 1455-1465, 2006.

34. Pillé JY, Denoyelle C, Varet J, Bertrand JR, Soria J, Opolon P, Lu H, Pritchard LL, Vannier JP and Malvy C: Anti-RhoA and anti-RhoC siRNAs inhibit the proliferation and invasiveness of MDA-MB-231 breast cancer cells in vitro and in vivo. Mol Ther 11: 267-274, 2005.
35. Vial E, Sahai E and Marshall CJ: ERK-MAPK signaling coordinately regulates activity of Rac1 and RhoA for tumor cell motility. Cancer Cell 4: 67-79, 2003.

36. Vasiliev JM, Omelchenko T, Gelfand IM, Feder HH and Bonder EM: Rho overexpression leads to mitosis-associated detachment of cells from epithelial sheets: A link to the mechanism of cancer dissemination. Proc Natl Acad Sci USA 101: 12526-12530, 2004.

37. Võsa U, Vooder T, Kolde R, Vilo J, Metspalu A and Annilo T: Meta-analysis of microRNA expression in lung cancer. Int $J$ Cancer 132: 2884-2893, 2013.

38. Guan P, Yin Z, Li X, Wu W and Zhou B: Meta-analysis of human lung cancer microRNA expression profiling studies comparing cancer tissues with normal tissues. J Exp Clin Cancer Res 31: $54,2012$.

39. Wang R, Wang ZX, Yang JS, Pan X, De W and Chen LB: MicroRNA-451 functions as a tumor suppressor in human non-small cell lung cancer by targeting ras-related protein 14 (RAB14). Oncogene 30: 2644-2658, 2011

40. He Q, Chen J, Lin HL, Hu PJ and Chen MH: Expression of peroxisome proliferator-activated receptor gamma, E-cadherin and matrix metalloproteinases-2 in gastric carcinoma and lymph node metastases. Chin Med J (Engl) 120: 1498-1504, 2007.

41. Inoue K, Kawahito Y, Tsubouchi Y, Yamada R, Kohno M, Hosokawa Y, Katoh D, Bishop-Bailey D, Hla T and Sano H: Expression of peroxisome proliferator-activated receptor (PPAR)-gamma in human lung cancer. Anticancer Res 21: 2471-2476, 2001

42. Tsubouchi Y, Sano H, Kawahito Y, Mukai S, Yamada R, Kohno M, Inoue K, Hla T and Kondo M: Inhibition of human lung cancer cell growth by the peroxisome proliferator-activated receptor-gamma agonists through induction of apoptosis. Biochem Biophys Res Commun 270: 400-405, 2000

43. Takamochi K, Ogura T, Yokose T, Ochiai A, Nagai K, Nishiwaki Y, Suzuki K and Esumi H: Molecular analysis of the TSC1 gene in adenocarcinoma of the lung. Lung Cancer 46: 271-281, 2004.

44. Liang MC, Ma J, Chen L, Kozlowski P, Qin W, Li D, Goto J, Shimamura T, Hayes DN, Meyerson M, et al: TSC1 loss synergizes with KRAS activation in lung cancer development in the mouse and confers rapamycin sensitivity. Oncogene 29: 1588-1597, 2010

45. Yang Z, Chen S, Luan X, Li Y, Liu M, Li X, Liu T and Tang H: MicroRNA-214 is aberrantly expressed in cervical cancers and inhibits the growth of HeLa cells. IUBMB Life 61: 1075-1082, 2009.

46. Zhang XJ, Ye H, Zeng CW, He B, Zhang $\mathrm{H}$ and Chen YQ: Dysregulation of miR-15a and miR-214 in human pancreatic cancer. J Hematol Oncol 3: 46, 2010.

47. Ueda T, Volinia S, Okumura H, Shimizu M, Taccioli C, Rossi S, Alder H, Liu CG, Oue N, Yasui W, et al: Relation between microRNA expression and progression and prognosis of gastric cancer: A microRNA expression analysis. Lancet Oncol 11: 136-146, 2010

48. Yanaihara N, Caplen N, Bowman E, Seike M, Kumamoto K, Yi M, Stephens RM, Okamoto A, Yokota J, Tanaka T, et al: Unique microRNA molecular profiles in lung cancer diagnosis and prognosis. Cancer Cell 9: 189-198, 2006.

49. Ishimura $M$, Sakurai-Yageta $M$, Maruyama $T$, Ando $T$, Fukayama M, Goto A and Murakami Y: Involvement of miR-214 and miR-375 in malignant features of non-small-cell lung cancer by down-regulating CADM1. J Cancer Ther 3: 379-387, 2012. 